\title{
Quelle politique régionale en contexte d'austérité?
}

\author{
Guy Chiasson \\ Université du Québec en Outaouais \\ Centre de recherche sur le développement
}

\section{INTRODUCTION}

\section{Pour leur part, les critiques ont souvent signalé la contribution importante des institutions régionales tant au niveau de l'emploi que de l'appui au potentiel de développement régional et ont fait valoir la volonté des acteurs régionaux de participer à la prise de décision sur les régions.}

Les réformes tous azimuts apportées par le gouvernement libéral $\mathrm{du}$ premier ministre Philippe Couillard ont généré bon nombre de commentaires, de critiques et de débats dans les médias et bien d'autres espaces publics de la société québécoise. Les réformes apportées aux mesures de soutien au développement des régions, en particulier l'abolition des Conférences régionales des élus (CRÉ) et le renforcement du lien d'autorité des MRC sur les Centres locaux de développement (CLD), n'ont pas fait exception là-dessus. Suite à l'annonce de ces mesures à l'automne 2014, le mouvement «Touche pas à ma région» a été créé pour dénoncer ces réformes. Ce mouvement, s'il n'a pas réussi à bloquer l'adoption du projet de loi 28 qui officialisait ces deux changements au développement régional, a tout de même su en faire un enjeu sur la place publique et retenir l'attention des médias pour une bonne partie de l'hiver et du printemps 2015 , y compris pour un temps celle des médias à l'échelle de l'ensemble du Québec. Le débat autour de ces mesures a surtout porté sur leur impact sur le développement des régions. D'un côté, les porte-paroles $\mathrm{du}$ gouvernement faisaient valoir que ces réformes permettaient d'éliminer du gaspillage de fonds publics dans des structures bureaucratiques. Les propos du premier ministre Couillard : «[c]'est qu'au lieu de se concentrer sur les missions comme le développement régional et le choix des projets par les régions et dans les régions, on se concentre sur les structures et leur financement avec les résultats qu'on a vus » (Gagnon, 2015) résument assez bien le justificatif répété maintes fois par les ténors du gouvernement. Pour leur part, les critiques ont souvent signalé la contribution importante des institutions régionales tant au niveau de l'emploi que de l'appui au potentiel de développement régional et ont fait valoir la volonté des acteurs régionaux de participer à la prise de décision sur les régions. À titre d'exemple, un texte signé par une cinquantaine d'universitaires québécois et publié dans Le Devoir qui soutient :

En ce sens, les directions régionales, Agences, Conseil régional des élus et autres instances publiques de planification sont des joueurs de premier plan pour la réussite du Québec. Abolir des postes dans la fonction publique, quand ce n'est pas l'institution complète, apparaît une visée à courte vue. C'est nier leur rôle dynamique et constructif dans les processus de développement. C'est nier qu'elles sont des acteurs de changement essentiel, porteurs d'expertises et de savoirs, qui permettent entre autres d'ajuster des programmes nationaux aux spécificités des territoires d'intervention. Bref, tuer nos institutions publiques, c'est tuer une ressource stratégique du développement des régions du Québec, que nous aurons mis des années à construire. (Fortin et Jean, 2014)

Les CRÉ et les CLD soumis au traitement de choc du gouvernement faisaient partie d'un ensemble plus large de moyens que l'État québécois déploie pour soutenir explicitement le développement régional. On peut considérer cet ensemble comme étant la politique de développement régional. Dans ce texte, je prendrai un point de vue un peu différent de celui qui a prédominé dans les médias. Je chercherai moins à identifier les 
impacts de ces mesures sur les régions et leur développement que de situer les réformes apportées par le gouvernement Couillard comme un changement dans les façons de faire au niveau de la politique publique en matière de développement régional. Dans quelle mesure ces réformes signifient un changement d'approche par rapport à la politique régionale qui a prévalu au Québec? Pour ce faire, je vais tout d'abord rappeler les grandes lignes de l'approche de développement régional québécoise pour ensuite situer les changements récents. Plus précisément, j'explorerai deux voies de changements majeurs : la municipalisation du développement et l'abandon du rôle d'accompagnateur de l'État.

\section{UN MODÈLE QUÉBÉCOIS DE DÉVELOPPEMENT RÉGIONAL}

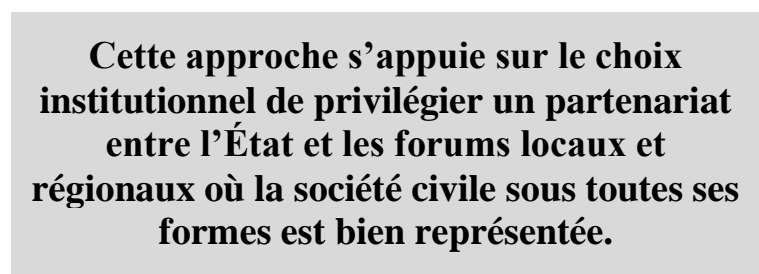

Il y a relativement peu de travaux scientifiques qui permettent de cerner l'approche de l'État québécois en matière de développement régional. Généralement, ces travaux émergent au moment où des réformes importantes de la politique régionale sont annoncées. Juan-Luis Klein (1995), en prenant comme point de repère l'importante réforme du développement régional de 1992, va situer cette réforme comme un cas de figure du passage de « l'État providence à l'État accompagnateur ». Cette réforme signifierait un changement au niveau de la responsabilité du développement. Si, dans une logique d'État providence, l'État était le grand responsable de la lutte aux disparités régionales, dans une logique d'État accompagnateur, l'État partage la responsabilité du développement des territoires avec des acteurs régionaux. Dans les mots de Klein (1995, p. 133) :

«le gouvernement abolit la plupart des dispositifs gouvernementaux créés depuis les années 1960 dans le but d'administrer une politique volontariste d'intervention régionale et il les remplace par d'autres dispositifs, ancrés dans la société civile dont la mission est d'assurer une planification régionale et partenariale du développement économique ».

Ce texte publié en 1995 annonçait déjà deux caractéristiques -un rôle de l'État comme partenaire et la présence de «dispositifs, ancrés dans la société civile »- que d'autres par la suite vont considérer comme des éléments centraux et distinctifs de l'approche québécoise en matière de développement des territoires et des régions. Les travaux de Benoît Lévesque (2004) situent le développement régional dans le cadre de ce qu'il qualifie de modèle québécois de développement. En parlant d'un «modèle québécois », Lévesque suggère que, depuis les années 1980, les diverses interventions québécoises en matière de développement régional ont suivi une approche relativement cohérente et comparable à ce qui se passe dans d'autres secteurs d'activité. Cette approche s'appuie sur le choix institutionnel de privilégier un partenariat entre l'État et les forums locaux et régionaux où la société civile sous toutes ses formes est bien représentée. Comme nous pourrons le voir dans les pages suivantes, les réformes annoncées par le gouvernement Couillard s'attaquent de front à au moins deux facettes de cette approche québécoise, soit la reconnaissance de la société civile comme un partenaire privilégié et la redéfinition du rôle de l'État comme un «accompagnateur». Cette analyse suggère que ces réformes qui se mettent en place participent à un renouvellement significatif, même si pas irrémédiable, de la politique de développement régional québécoise.

\section{LA MUNICIPALISATION DU DÉVELOPPEMENT}

Tel que mentionné plus haut, une des caractéristiques importantes de l'approche québécoise était de s'appuyer sur un ensemble de forums locaux et régionaux qui devenaient les interlocuteurs (ou partenaires) privilégiés de l'État dans le cadre de la politique régionale. Ces 
forums étaient créés aux fins du développement local et régional et étaient volontairement distincts de la structure municipale (municipalités locales, MRC), même si les élus étaient appelés à y participer en compagnie d'acteurs représentants de la société civile. Les réformes du gouvernement ont démontré une volonté claire de mieux intégrer le développement régional dans le giron municipal.

À l'échelle régionale, le gouvernement a carrément aboli les Conférences régionales des élus (CRÉ), transférant par la même occasion les budgets de développement régional administrés par les CRÉ et leur rôle d'interlocuteur régional de l'État québécois vers les Municipalités régionales de comtés (MRC). Par la suite, d'autres instances actives à l'échelle régionale ne seront pas épargnées et cela sans que ne soit précisé qui va exercer leur mandat. C'est le cas pour les Commissions régionales des ressources naturelles et du territoire, instance de planification concertée du territoire public qui relevait des CRÉ, et des Forums Jeunesse récemment dissolus par le gouvernement. Si on ajoute à cela la très récente annonce par le gouvernement de son intention de fusionner les onze Coopératives de développement régional en une seule grande coopérative québécoise, il se dégage une très nette impression que le gouvernement accorde désormais assez peu d'importance au palier régional comme un espace structurant pour l'action publique et la représentation de la société civile.

\section{La disparition des CRÉ, le rétrécissement de l'importance du palier régional et l'intégration des CLD démontrent assez clairement une volonté de remettre le développement dans les mains des élus locaux et de mieux le « localiser » dans le pouvoir municipal.}

À l'échelle des MRC, la réforme prévoit que les MRC vont intégrer le mandat de développement local des CLD, ce qui en pratique veut dire que chaque conseil de MRC pourra décider d'absorber son CLD ou de le maintenir comme entité distincte. Un nombre important de MRC, faisant face à des coupures importantes dans les diffé- rentes enveloppes gouvernementales transférées au CLD, ont choisi d'intégrer ces derniers, de sorte que dans bien des régions, la présence de CLD autonomes est en damier et du même coup le réseau qui chapeautait ces CLD, l'Association des centres locaux de développement du Québec, a été aboli. Un document distribué aux participants lors du Forum Développer ensemble nos territoires, précisait que :

selon les informations disponibles, environ $1 / 3$ des CLD auront disparu au cours des prochaines semaines et leur mandat de soutien aux entreprises sera repris par les MRC. Ailleurs, les décisions n'ont pas encore été prises et cette proportion pourrait donc être plus importante dans un an. À Montréal, 6 nouvelles organisations apparaissent et prendront graduellement la place des 18 CLD-CDEC antérieurs. La place de représentants de la société civile y sera vraisemblablement plus faible (TIESS, 2015).

La disparition des CRÉ, le rétrécissement de l'importance du palier régional et l'intégration des CLD démontrent assez clairement une volonté de remettre le développement dans les mains des élus locaux et de mieux le «localiser » dans le pouvoir municipal. C'est tout à fait dans le sens de ce que Jean-François Simard et Yvon Leclerc (2008) qualifient de «municipalisation du développement ». Il faut bien admettre, à l'instar de ces auteurs, que ce travail d'arrimage du développement régional au pouvoir municipal avait déjà été bien amorcé en 2003 par le gouvernement Charest. Ce dernier avait alors changé la composition des Conseils régionaux de développement (CRD), devenus les CRÉ suite à la réforme, pour faire une place plus importante aux élus locaux et au détriment des représentants de la société civile. La loi prévoyait également un rapprochement entre les CLD et les MRC. Ces changements cherchaient à miser sur l'imputabilité des élus locaux en renforçant leur autorité sur le développement local et régional. À l'époque plusieurs s'étaient prononcés contre l'exclusion de la société civile de la structure de gouvernance des CRÉ et des CLD (Simard et Leclerc, 2008; Lévesque, 2004) y voyant une perte pour le développement régional. Face à la disparition annoncée du gouvernement, ces critiques doivent maintenant défendre les CRÉ et 
les CLD autonomes et ce qu'il reste d'espace pour la participation de la société civile. C'est donc dire que les plus récentes réformes du gouvernement Couillard ont repris la municipalisation du développement là où le gouvernement Charest l'avait laissé en 2003-2004 et l'a poussé jusqu'au bout. La MRC devient ainsi le partenaire principal $\mathrm{du}$ gouvernement québécois en matière de développement du territoire, alors que la société civile et le palier régional voient leur espace d'influence se réduire comme une peau de chagrin.

\section{LE PACTE FISCAL TRANSITOIRE OU L'APPUI TIMORÉ AU DÉVELOPPEMENT RÉGIONAL}

\section{L'approche québécoise de développement régional s'appuie sur un rôle de l'État comme partenaire des milieux régionaux et locaux.}

Dans la section précédente, ma présentation des réformes apportées par le gouvernement s'est limitée à l'abolition des CRÉ et le renforcement de l'emprise des MRC sur les CLD, c'est-à-dire celles qui ont été le plus discutées et débattues sur la place publique. Comme nous avons pu le voir, ces mesures signifiaient un important glissement vers les municipalités comme principal partenaire de l'État et comme échelle d'action publique en matière de développement régional. Alors que l'attention s'est concentrée sur le transfert de pouvoir de la société civile vers les municipalités, le débat public a été moins virulent sur un autre versant de la politique régionale, soit le rôle de l'État comme partenaire. Là aussi, comme on pourra le voir, les réformes du gouvernement Couillard se distancient de l'approche précédente de politique régionale au Québec.

L'approche québécoise de développement régional s'appuie sur un rôle de l'État comme partenaire des milieux régionaux et locaux. Dans les mots de Benoît Lévesque (2004, p. 11), dans le contexte du modèle québécois des années 1980 et 1990 :

L'État joue un rôle d'animateur et de catalyseur favorisant la multiplication des ententes entre partenaires économiques et non-économiques et fournissant le soutien pour les outils et les apprentissages collectifs nécessaires. Par conséquent, contrairement aux idées reçues, l'intervention de l'État dans l'économie n'a pas disparu, elle s'est maintenue voire accrue, mais selon des modalités différentes de celle de la Révolution tranquille et de l'inspiration keynésienne.
Il donne plusieurs exemples pour montrer comment l'État assure concrètement ce rôle de soutien au dynamisme des acteurs du territoire : la politique industrielle, les Fonds d'investissement, etc.). À cette liste, on pourrait également ajouter d'autres initiatives comme le programme Action concertée de coopération régionale de développement (ACCORD) ou la Politique nationale de la ruralité (Jean, 2003) mis en place directement dans le cadre de la politique de développement régional. Ces derniers programmes démontrent, non seulement une volonté de l'État d'appuyer les milieux dynamiques dans leur quête de développement, mais aussi d'offrir des moyens à des milieux plus dévitalisés pour se développer.

À bien des égards, les réformes Couillard semblent représenter un recul par rapport à la volonté de l'État québécois d'assurer son rôle de «catalyseur », en particulier auprès des milieux plus dévitalisés. Ce recul est particulièrement observable, il me semble, dans le cadre du pacte fiscal transitoire. Rappelons que le pacte fiscal c'est l'entente financière qui lie les municipalités locales, les MRC et le gouvernement. C'est dans le cadre de ce pacte fiscal que le gouvernement s'engage au niveau des transferts monétaires vers les pouvoirs locaux. Dans un contexte où les municipalités et MRC sont appelées à jouer un rôle croissant en matière de développement des territoires, le pacte fiscal devient un bon indicateur des moyens concédés par l'État pour leurs responsabilités en matière de développement.

Le pacte fiscal transitoire représente une importante réduction de moyens par rapport à ce que recevaient auparavant les municipalités et les MRC. André Joyal, dans un autre article de ce numéro, rappelle que ces coupes seront de l'ordre de $10 \%$ (300 millions de dollars). Cette coupure est très substantielle surtout si l'on tient compte de la ponction supplémentaire de 40 millions de dollars 
(55\%) dans les enveloppes auparavant destinées aux CLD et maintenant gérées en bonne partie par les MRC.

Dans le cadre d'un pacte fiscal «transitoire » à renégocier sous peu, il est difficile de prévoir avec grande certitude comment les choses vont évoluer dans les prochaines ententes entre le gouvernement et les MRC. Il demeure que les coupes inscrites dans le pacte signifient une diminution très appréciable des moyens sur lesquels pourront compter les municipalités pour prendre en charge leurs différentes responsabilités. Il faut dire que le gouvernement a fait valoir à quelques reprises qu'il comptait refinancer le municipal via un nouveau partage des redevances des ressources naturelles (Lafleur, 2015). Par contre, pour l'instant les termes et les montants de ce nouveau partage restent inconnus, ce qui rend très difficile d'apprécier son impact sur la prise en charge municipale du développement des territoires.

Il faut se rappeler que les municipalités québécoises, comme celles des autres provinces canadiennes, ont longtemps concentré leur action leur action sur ce que d'aucuns qualifient de « service à la propriété » (Andrew, 1999), ce qui veut dire surtout la mise en place et l'entretien d'infrastructures (eau, égout, etc.) et de service de base (vidange, déblayage de la neige, pompiers, etc.) et l'aménagement du territoire dans le cas des MRC. Si des responsabilités comme le développement social ou encore le développement local ne sont pas totalement nouvelles pour les municipalités (Letarte, 2003), elles ont tendance à se rajouter à ces responsabilités de base et à nécessiter des apprentissages et de nouvelles expertises de la part

\section{CONCLUSION}

\section{On pourrait bien penser que ces mesures s'inscrivent dans un effort plus ou moins explicite de changer les façons de faire le développement régional et donc que derrière ces changements se dissimulerait une nouvelle politique de développement régional.}

L'objectif de ce texte était de voir dans quelle mesure la série de changements apportés par le gouvernement Couillard participe à la construction d'une nouvelle politique de développement des municipalités. Dans ce contexte, on peut se demander si la diminution des transferts provinciaux vers les municipalités ne va pas favoriser un recentrement, au moins de certaines municipalités, sur leur métier de base, c'est-à-dire sur le service à la propriété. À tout le moins, on peut s'imaginer que pour bon nombre de municipalités, il sera difficile d'intégrer de nouvelles responsabilités en matière de développement régional, alors que les budgets transférés par Québec, en particulier ceux dédiés au développement local, sont revus substantiellement à la baisse.

J'argumenterai que le pacte fiscal actuel contribue de surcroît à accentuer des inégalités entre les milieux que les politiques précédentes tentaient d'amenuiser. Les plus grandes municipalités urbaines qui ont accès à une richesse foncière relativement importante sont passablement moins dépendantes sur les transferts gouvernementaux pour prendre des nouvelles responsabilités en matière de développement régional. C'est bien différent pour bien des petites municipalités rurales pour qui les fonds provenant de Québec sont essentiels pour assurer une certaine action en matière de développement régional. Cette nette impression que le gouvernement se désengage envers le principe de l' « occupation dynamique du territoire » et les municipalités dévitalisées est fortement renforcée par le fait que le gouvernement a abandonné la Politique nationale de la ruralité et n'a pas renouvelé le Plan d'action en appui aux municipalités dévitalisées (Vachon, 2015), tous deux des mesures qui donnait des moyens d'actions aux municipalités locales et les MRC dans des contextes de défavorisation.

régional. Plus précisément, mon analyse a porté sur trois mesures explicitement dédiées au développement régional -l'abolition des CRÉ, l'intégration des CLD dans le giron des MRC et le pacte fiscal transitoire- laissant ainsi de côté certaines mesures sectorielles comme la réforme des structures régionales en santé ou l'abolition de bureaux régionaux de certains ministères et d'autres mesures comme l'abolition de la Politique nationale de la ruralité et la fin du financement accordé à Solidarité rurale qui ont tous des impacts certains sur les dynamiques régionales. L'analyse de ces trois 
changements montre qu'elles sont en rupture assez catégorique avec l'approche qui s'était imposée depuis les années 1980 et que certains ont qualifiée de modèle québécois de développement. En donnant aux pouvoirs locaux élus un rôle central dans la mise en œuvre de la politique de développement régional et en diminuant les fonds rendus disponibles pour les municipalités, le gouvernement modifie significativement la relation partenariale qui était au cœur de l'approche québécoise. De surcroît, en tournant le dos à l'échelle régionale, le gouvernement modifie le terrain de jeu du développement régional.

Cela suggère que le programme d'austérité du gouvernement Couillard ne se limite pas, comme c'est présenté par certains, à un exercice comptable visant à diminuer les dépenses de l'État. On pourrait bien penser que ces mesures s'inscrivent dans un effort plus ou moins explicite de changer les façons de faire le développement régional et donc que derrière ces changements se dissimulerait une nouvelle politique de développement régional. Cette politique ferait porter plus lourdement le poids du développement régional sur les pouvoirs locaux, non seulement en laissant beaucoup moins de place à des forums de la société civile, mais aussi en limitant la contribution de l'État comme partenaire. Le risque fort que fait peser cette approche, si elle se maintient dans les pactes fiscaux à venir, et l'avenir le dira sans doute assez rapidement, c'est celui d'une certaine métropolisation du développement régional au Québec. L'idée de métropolisation (Veltz, 1999) renvoi à un développement de plus en plus concentré dans les espaces métropolitains parce que ces milieux centraux sont les seuls qui peuvent réunir les moyens stratégiques pour faire face aux nouvelles exigences de la globalisation. Veltz parle ainsi d'une «économie d'archipel » alors les territoires situés à l'extérieur des axes métropolitains seraient en quelque sorte condamnés à la marginalité faute de moyens de porter leur propre développement. On peut bien relativiser le caractère mécanique de cette logique, mais il reste qu'en faisant porter de plus en plus le développement sur les municipalités, le gouvernement québécois abdique une certaine responsabilité dans le rééquilibrage des moyens auxquels les milieux locaux peuvent faire appel pour se développer. Il risquerait ainsi de contribuer à renforcer un «Québec cassé en deux », déjà dénoncé par le Conseil des affaires sociales (Leclerc, 1989) à la fin des années 1980.

\section{BIBLIOGRAPHIE ET NOTES}

Andrew, Caroline (1999). «Les métropoles» dans Caroline Andrew (s.d.) Dislocation et permanence. L'invention du Canada au quotidien, Ottawa, Presses de l'Université d'Ottawa, p. 61-79.

Fortin, Marie-José et Bruno Jean (2014). «Le Québec privé de ses joueurs de premier plan. Abolir des institutions de l'administration publique régionale relève d'une visée à courte vue », Le Devoir, 5 novembre, en ligne http:// www.ledevoir.com/politique/quebec/422920/developpem ent-regional-agences-cre-cld-des-joueurs-de-premier-plan Gagnon, Mélyssa (2015). « Philippe Couillard est las du discours pessimiste », Le Droit, 2 juin, p. 13.

Klein, Juan-Luis (1995). «De l'État-providence à l'État accompagnateur dans la gestion du social: le cas du développement régional au Québec », Lien social et politiques, no 33, p. 133-141.

Lafleur, Claude (2015). «Pierre Moreau prêt à poursuivre la décentralisation. Le ministre s'attend à des 'efforts additionnels' ", Le Devoir, 16 mai, en ligne http://www.ledevoir.com/politique/quebec/439976/pierr e-moreau-pret-a-poursuivre-la-decentralisation-le-

ministre-s-attend-a-des-efforts-additionnels

Leclerc, Yvon (1989), Deux Québec dans un: rapport sur le développement social et démographique, Conseil des Affaires sociales, Gouvernement du Québec, Québec, 124 pages.

Letarte, Georges (2003). Les municipalités et le développement social. Un cas-type : les municipalités de la région de la Capitale-Nationale, Rimouski, GRIDEQ. Lévesque, Benoît (2004). «Le développement local et régional : vers la fin du modèle québécois? », Cahiers de l'ARUC en économie sociale I-03-2004. Disponible en ligne http://www.aruces.uqam.ca/Portals/0/ cahiers/I-032004.pdf

Simard, Jean-François et Yvon Leclerc (2008). «Les Centres locaux de développement 1998-2008. Une gouvernance en mutation: entre participation citoyenne et imputabilité municipale», Revue canadienne des sciences régionales, volume 31, numéro 3, pp. : 615-636. Territoires Innovants en économie sociale et solidaire (TIESS) (2015). Aperçu des transformations dans le développement territorial, document distribué dans le cadre du Forum Développer ensemble nos régions, Nicolet, 13 mai.

Vachon, Bernard (2015). « 10 éléments de réflexion au Ministre Moreau », Néorural, 25 mars, en ligne http://neorurale.ca/2015/03/10elementsdereflexionm inistremoreau/

Veltz Pierre, (1996), Mondialisation, villes et territoires : l'économie d'archipel, Paris, PUF. 